\title{
Vibration Analysis of Axially Functionally Graded Tapered Euler-Bernoulli Beams Based on Chebyshev Collocation Method
}

\author{
Wei-Ren Chen \\ Chinese Culture University, Taipei 11114, Taiwan.
}

(Received 3 January 2020; accepted 13 July 2020)

\begin{abstract}
The bending vibration behavior of a non-uniform axially functionally graded Euler-Bernoulli beam is investigated based on the Chebyshev collocation method. The cross-sectional and material properties of the beam are assumed to vary continuously across the axial direction. The Chebyshev differentiation matrices are used to reduce the ordinary differential equations into a set of algebraic equations to form the eigenvalue problem associated with the free vibration. Some calculated results are compared with numerical results in the published literature to validate the accuracy of the present model. A good agreement is observed. The effects of the taper ratio, volume fraction index, and restraint types on the natural frequency of axially functionally graded beams with non-uniform cross section are examined.
\end{abstract}

\section{INTRODUCTION}

Functionally graded (FG) materials are special composites with smoothly varying material properties along any desired spatial direction. These graded properties can be achieved by gradually changing the volume fraction of constituents along a typical direction according to the polynomial, power, and exponential laws. Due to these particular graded properties, the applications of structures with FG materials have received wide attention in civil, automobile and aerospace industries for the past few decades. Because a beam is both a commonly and widely used member in structures and machines, to better understand the dynamic behavior of a beam made of FG materials is necessary from a structural design point of view. In past years, the dynamic behaviors of FG beams with material properties varying along the beam thickness have been thoroughly investigated by many researchers using various analytical and numerical methods. ${ }^{1-12}$ It is well-known that beam structures with varying cross-sectional and material properties along the length direction are commonly used in buildings, bridges and mechanical components due to the fact that they are capable of optimizing the strength and weight of the structure. Hence, it is important to accurately predict and evaluate the dynamic characteristics of such beam structures. In this regard, only the literature related to the FG beams with axially varying properties will be discussed next.

Due to the variable coefficients in the governing equation, the dynamic analysis of axially FG beams with tapered crosssections becomes more mathematically complex. Therefore, the dynamic problems of FG beams with material and crosssectional properties varying along the axial direction have been largely studied by numerical methods based on various beam theories. Aydogdu investigated the vibration and buckling analysis of simply supported FG Euler-Bernoulli beams with axial variation of material properties based on a semi-inverse method. ${ }^{13}$ Huang and Li applied the integral equation method to study the free vibration of non-uniform axially FG EulerBernoulli beams. ${ }^{14}$ The effects of material graded parameters on natural frequencies of the beams were investigated. Based on the finite element method, Shahba et al. dealt with the free vibration and stability of axially FG tapered Timoshenko beams with respective classic and non-classical boundary conditions. ${ }^{15}$ Hein and Feklistova studied the free vibration of non-uniform axially FG beams using the Euler-Bernoulli beam theory and Haar wavelet approach. ${ }^{16}$ The results revealed that the Haar wavelet approach was capable of calculating the frequencies of beams with different non-uniform cross-sections, bending rigidity and mass density. Shahba and Rajasekaran studied the free vibration and stability of axially FG tapered Euler-Bernoulli beams by using both the differential transform element method and differential quadrature element method of lowest-order. ${ }^{17}$ The free longitudinal and transverse natural frequencies, and the critical buckling loads, of the beams were determined by the two numerical methods. Li et al. presented the exact frequency equations of the free vibration for axially exponentially FG beams with different end conditions based on an analytical approach. ${ }^{18}$ Rajasekaran analysed the bending vibration of rotating axially FG tapered Euler-Bernoulli beams based on the differential transform element method and differential quadrature element method of lowest-order. ${ }^{19}$ The effects of material property, taper ratio, rotating speed, hub radius and tip mass on the natural frequencies were investigated. Huang et al. investigated the free vibration of non-uniform and axially FG Timoshenko beams with various boundary conditions by using a unified approach. ${ }^{20}$ Sarkar and Ganguli studied the free vibration of axially FG uniform Timoshenko beams with fixed-fixed boundary conditions. ${ }^{21}$ Tang et al. derived the exact frequency equations of the free transverse vibration of exponentially FG beams with non-uniform cross-section based on the Timoshenko beam theory. ${ }^{22}$ Liu et al. analysed the free bending vibration of axially FG Euler-Bernoulli beams with tapered cross-section using the spline finite point method. ${ }^{23}$ The effects of the material and cross-sectional properties varying along the axial direction on the natural frequencies were discussed. Cao and Gao investigated the free vibration of axially FG beams with non-uniform cross-section by using the asymptotic development method. ${ }^{24}$ 


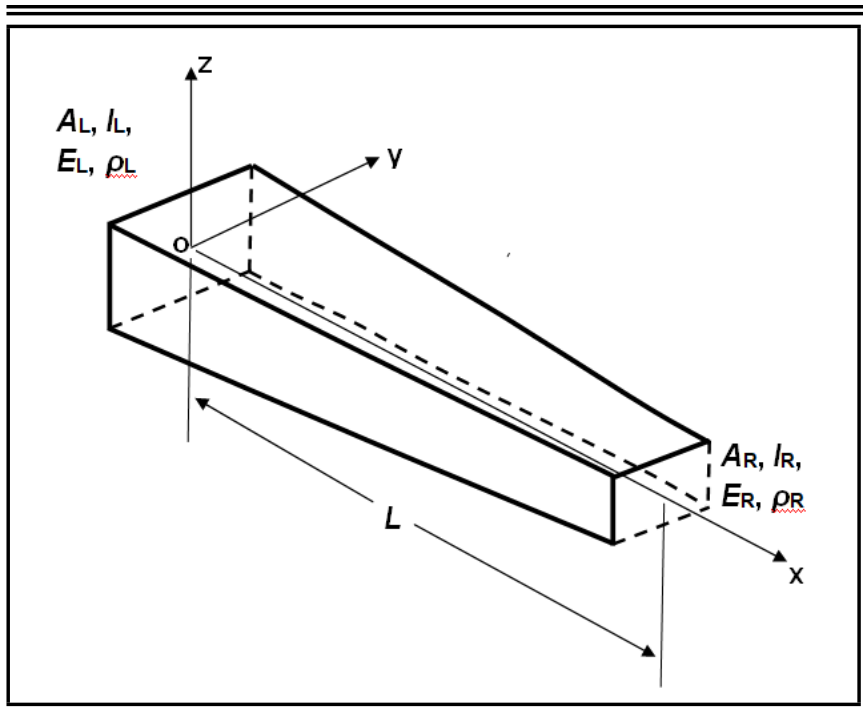

Figure 1. Configuration and coordinates of tapered axially FG beam.

As mentioned above, various analytical and numerical methods had been used to effectively investigate the influences of material and geometric parameters on the dynamic characteristics of axially FG beams. It is well-known that the Chebyshev collocation method has been applied to different mathematical and engineering models because of its high rate of convergence and predictable accuracy. ${ }^{8-10,12,25-34}$ However, the application of this method to the bending vibration of axially FG tapered beams has seldom been reported. Hence, the present paper attempts to study the free transverse vibration of the axially FG Euler-Bernoulli beam with a tapered cross-section based on the Chebyshev collocation method. The Chebyshev differentiation matrices are applied to transform the governing differential equations into a set of algebraic eigenvalue equations. The lateral natural frequencies of tapered axially FG beams with various boundary conditions are then obtained by solving the generalized eigenvalue equation. The material properties axially graded according to the exponential, polynomial and power functions are considered. The rectangular cross section is assumed to be tapered linearly in the width and height directions along the beam length. The effects of the taper ratios, axially graded properties, and boundary conditions on the free vibration behaviors of the axially FG tapered beams are investigated. Several numerical results are evaluated and compared with those in the published literature to validate the accuracy of the present model. The results reveal that the proposed method can be used to study the free vibration of tapered EulerBernoulli beams with different axially graded material properties described by typical functions under various boundary conditions with good accuracy.

\section{PROBLEM FORMULATION}

The present paper investigates the bending vibration behavior of non-uniform axially FG beams under various boundary conditions. The material and cross-section properties were assumed to be varied along the longitudinal direction of the beam. Figure 1 illustrates the geometric configuration and coordinate system of the beam with a tapered section along the height and width directions. The origin $o$ of the coordinate $x y z$ was the centre of the left-end plane of the beam. The $x$-axis, $y$-axis and $z$-axis originating from the origin were found in the length, width, and thickness directions, respectively. $A, I, E$ and $\rho$ represented the respective cross-sectional area, area moment of inertia, Young modulus, and density; the subscripts $o$ and $L$ denoted the axial position of the given properties at $x=0$ and $x=L$, respectively. The governing equation of motion of such a beam was expressed by a fourth-order partial differential equation as follows. ${ }^{14,17}$

$$
\frac{\partial^{2}}{\partial x^{2}}\left[S(x) \frac{\partial^{2} w(x, t)}{\partial x^{2}}\right]+m(x) \frac{\partial^{2} w(x, t)}{\partial t^{2}}=0, \quad 0 \leq x \leq L .
$$

Here $w$ was the transverse displacement, $L$ was the beam length, and $x$ was the axial coordinate. $S(x)=E(x) I(x)$ was the bending rigidity where $E(x)$ and $I(x)$ were the Young modulus and area moment of inertia at position $x$, respectively. $m(x)=\rho(x) A(x)$ was the beam mass per unit length in which $\rho(x)$ and $A(x)$ were the respective density and cross-sectional area at location $x$. Assume that $w(x, t)=W(x) e^{i \omega t}$ and substitute it into Eq. (1) to yield:

$$
\frac{\mathrm{d}^{2}}{\mathrm{~d} x^{2}}\left[S(x) \frac{\mathrm{d}^{2} W}{\mathrm{~d} x^{2}}\right]-m(x) \omega^{2} W=0, \quad 0 \leq x \leq L
$$

where $\omega$ was the natural frequency. Changing the spatial variables by $\xi=2 x / L-1$ and letting $\Omega^{2}=\omega^{2} L^{4} / 16$ allowed Eq. (2) to be rewritten as:

$$
\frac{\mathrm{d}^{2}}{\mathrm{~d} \xi^{2}}\left[S(\xi) \frac{\mathrm{d}^{2} W}{\mathrm{~d} \xi^{2}}\right]-m(\xi) \Omega^{2} W=0, \quad-1 \leq \xi \leq 1 ;
$$

or

$$
\begin{aligned}
& S(\xi) \frac{\mathrm{d}^{4} W}{\mathrm{~d} \xi^{4}}+2 S^{\prime}(\xi) \frac{\mathrm{d}^{3} W}{\mathrm{~d} \xi^{3}}+2 S^{\prime \prime}(\xi) \frac{\mathrm{d}^{2} W}{\mathrm{~d} \xi^{2}}=m(\xi) \Omega^{2} W \\
& -1 \leq \xi \leq 1
\end{aligned}
$$

The associated classic boundary conditions were represented as follows:

$$
\begin{aligned}
& W=0, \quad \frac{\mathrm{d} W}{\mathrm{~d} \xi}=0 \quad \text { (Clamped end) } \\
& W=0, \quad \frac{\mathrm{d}^{2} W}{\mathrm{~d} \xi^{2}}=0 \quad \text { (Pinned end) } \\
& \frac{\mathrm{d}^{2} W}{\mathrm{~d} \xi^{2}}=0, \quad \frac{\mathrm{d}}{\mathrm{d} \xi}\left[S(\xi) \frac{\mathrm{d}^{2} w}{\mathrm{~d} \xi^{2}}\right]=0 \quad \text { (Free end) }
\end{aligned}
$$

The free bending vibration problem of non-uniform axially FG beams in Eqs. (4) and (5) were solved using the Chebyshev collocation method. The following Gauss-Chebyshev-Lobatto collocation points within the interval $[-1,1]$ were used: ${ }^{35}$

$$
\xi_{j}=\cos \frac{\pi j}{N}, \quad j=0,1,2, \ldots, N
$$

Thus, the displacement function $W(\xi)$ was expanded by the Nth-order Chebyshev polynomials as follows:

$$
W(\xi) \approx \sum_{N}^{j=0} \gamma_{j}(\xi) W\left(\xi_{j}\right), \quad j=0,1,2, \ldots, N .
$$


with

$$
\begin{aligned}
& \gamma_{j}(\xi)=\frac{(-1)^{j+1}\left(1-\xi^{2}\right) T_{N}^{\prime}(\xi)}{c_{j} N^{2}\left(\xi-\xi_{j}\right)} ; \\
& T_{N}\left(\xi_{j}\right)=\cos \left(N \cos ^{-1}\left(\xi_{j}\right)\right) ; \\
& \gamma_{j}\left(\xi_{k}\right)=\delta_{j k} \\
& c_{j}=\left\{\begin{array}{ll}
2 & j=0, \quad N=0 \\
1 & j=1,2, \ldots, \quad N-1
\end{array} .\right.
\end{aligned}
$$

Then, the first derivative of the displacement function $W(\xi)$ was obtained by the following matrix vector multiplication:

$$
W^{\prime}\left(\xi_{j}\right)=\sum_{j=0}^{N}\left(D_{N}\right)_{i j} W\left(\xi_{j}\right), \quad j=0,1,2, \ldots, N
$$

Here $\left(D_{N}\right)_{i j}=\gamma_{j}^{\prime}\left(\xi_{j}\right)$ was the $i, j$ element of an $(N+1) \times$ $(N+1)$ Chebyshev differentiation matrix $D_{N}$. The entries of this matrix were: ${ }^{35}$

$$
\begin{aligned}
& \left(D_{N}\right)_{00}=\frac{2 N^{2}+1}{6} ; \quad\left(D_{N}\right)_{N N}=-\frac{2 N^{2}+1}{6} ; \\
& \left(D_{N}\right)_{j j}=-\frac{\xi_{j}}{2\left(1-\xi_{j}^{2}\right)}, \quad j=1,2, \ldots, N-1 ; \\
& \left(D_{N}\right)_{i j}=\frac{c_{i}(-1)^{i+j}}{c_{j}\left(\xi_{i}-\xi_{j}\right)}, \quad i \neq j, \quad j=1,2, \ldots, N-1 .
\end{aligned}
$$

For simplicity, the first derivative of the Chebyshev differentiation matrix was denoted by $D_{1}$. The kth derivative was obtained by $D_{k}=\left(D_{1}\right)^{k}$.

Based on the Chebyshev collocation method as described, the ordinary differential equation in Eq. (4) was rewritten in terms of Chebyshev differentiation matrices as:

$$
\left(K_{1} D_{4}+2 K_{2} D_{3}+K_{3} D_{2}\right) \bar{W}=\Omega^{2} M \bar{W} ;
$$

where

$$
\begin{aligned}
K_{1}= & {\left[\begin{array}{cccc}
S\left(\xi_{0}\right) & 0 & \ldots & 0 \\
0 & S\left(\xi_{1}\right) & \ldots & 0 \\
\vdots & \vdots & \ddots & \vdots \\
0 & 0 & \ldots & S\left(\xi_{N}\right)
\end{array}\right] ; } \\
K_{2}= & {\left[\begin{array}{cccc}
S^{\prime}\left(\xi_{0}\right) & 0 & \ldots & 0 \\
0 & S^{\prime}\left(\xi_{1}\right) & \ldots & 0 \\
\vdots & \vdots & \ddots & \vdots \\
0 & 0 & \ldots & S^{\prime}\left(\xi_{N}\right)
\end{array}\right] ; } \\
K_{3}= & {\left[\begin{array}{cccc}
S^{\prime \prime}\left(\xi_{0}\right) & 0 & \ldots & 0 \\
0 & S^{\prime \prime}\left(\xi_{1}\right) & \ldots & 0 \\
\vdots & \vdots & \ddots & \vdots \\
0 & 0 & \ldots & S^{\prime \prime}\left(\xi_{N}\right)
\end{array}\right] ; } \\
m= & {\left[\begin{array}{cccc}
m\left(\xi_{0}\right) & 0 & \ldots & 0 \\
0 & m\left(\xi_{1}\right) & \ldots & 0 \\
\vdots & \vdots & \ddots & \vdots \\
0 & 0 & \ldots & m\left(\xi_{N}\right)
\end{array}\right] ; } \\
\bar{W}= & \left\{W\left(\xi_{0}\right) W\left(\xi_{1}\right) \ldots W\left(\xi_{N}\right)\right\}^{T}=\left\{W_{1} W_{2} \ldots W_{N+1}\right\}^{T} .
\end{aligned}
$$

Likewise, the boundary equations in Eq. (5) at the supporting ends of the beam were also expressed by Chebyshev differentiation matrices as given in Table 1 . When the homogeneous boundary conditions were imposed on the governing Eq. (11), some mathematical operations were performed. First, the first and second equations of the system were replaced by the two boundary conditions at the right end. The $N$ th and $(N+1)$ th equations were replaced by those at the left end. Then, the $N$ th and $(N+1)$ th equations of the new system were moved up to become the third and fourth equations. Finally, by shifting the displacements $W_{N}$ and $W_{N+1}$ to the third and fourth rows of the displacement vector $\bar{W}$, the generalized eigenvalue problem was obtained as:

$$
\begin{aligned}
& {\left[\begin{array}{cc}
K_{B B} & K_{B I} \\
K_{I B} & K_{I I}
\end{array}\right]\left\{\begin{array}{l}
\overline{W_{B}} \\
\overline{W_{I}}
\end{array}\right\}=\Omega^{2}\left[\begin{array}{cc}
O & O \\
M_{I B} & M_{I I}
\end{array}\right]\left\{\begin{array}{l}
\overline{W_{B}} \\
\overline{W_{I}}
\end{array}\right\} ; } \\
& \overline{W_{B}}=\left\{\begin{array}{llll}
W_{1} & W_{2} & W_{N} & W_{N+1}
\end{array}\right\}^{T} \\
& \overline{W_{I}}=\left\{\begin{array}{llll}
W_{3} & W_{4} & \ldots & W_{N-1}
\end{array}\right\}^{T} .
\end{aligned}
$$

The subscripts B and I denoted the boundary and internal collocation points associated with the boundary condition and the governing equation, respectively. The sizes of the stiffness matrices $K_{B B}, K_{B I}, K_{I B}$ and $K_{I I}$ were $4 \times 4,4 \times(N-3)$, $(N-3) \times 4$ and $(N-3) \times(N-3)$, respectively. The sizes of the inertia matrices $M_{I B}$ and $M_{I I}$ were the same as the corresponding stiffness matrices $K_{I B}$ and $K_{I I}$.

To solve the general eigenvalue equation in Eq. (13), it was expanded to yield:

$$
\begin{gathered}
K_{B B} \overline{W_{B}}+K_{B I} \overline{W_{I}}=0 \\
K_{I B} \overline{W_{B}}+K_{I I} \overline{W_{I}}=\Omega^{2}\left(M_{I B} \overline{W_{B}}+M_{I I} \overline{W_{I}}\right) .
\end{gathered}
$$

Then, after introducing Eq. (15) into Eq. (16), the algebraic eigenvalue equation was reduced to the following form:

$$
A \overline{W_{I}}=\Omega^{2} B \overline{W_{I}} \text {; }
$$

$$
\begin{aligned}
& A=-K_{I B}\left(K_{B B}\right)^{-1} K_{B I}+K_{I I} \\
& B=-M_{I B}\left(K_{B B}\right)^{-1} K_{B I}+M_{I I} .
\end{aligned}
$$

Thus, natural frequencies of the free bending vibration of nonuniform axially FG Euler-Bernoulli beams with various classical boundary conditions can be obtained as the solution:

$$
\operatorname{det}\left(A-\Omega^{2} B\right)=0 .
$$

\section{RESULTS AND DISCUSSIONS}

To assure the successful application of the Chebyshev collocation method in the vibration analysis of axially FG EulerBernoulli beams with non-uniform cross-sections, the accuracy studies are carried out through various numerical examples. First example to be concerned is a uniform axially FG beam with the bending rigidity $S(x)$ and mass per unit length $m(x)$ according to the following distribution: ${ }^{14}$

$$
\begin{aligned}
& S(x)=\left[E_{o}+\left(E_{L}-E_{o}\right) \frac{e^{\alpha x / L}-1}{e^{\alpha}-1},\right] I \quad \alpha \neq 0 \\
& m(x)=\left[\rho_{o}+\left(\rho_{L}-\rho_{o}\right) \frac{e^{\alpha x / L}-1}{e^{\alpha}-1}\right] A, \quad \alpha \neq 0
\end{aligned}
$$


Table 1. Boundary condition equations in terms of Chebyshev differentiation matrices.

\begin{tabular}{|c|c|c|}
\hline Boundary Conditions & Left end $(\xi=-1)$ & Right end $(\xi=+1)$ \\
\hline clamped & $\begin{array}{l}{\left[\begin{array}{lllr}0 & 0 & \cdots & 1\end{array}\right] \bar{W}=0} \\
{\left[D_{1}(N+1,:)\right] \bar{W}=0}\end{array}$ & $\begin{array}{l}{\left[\begin{array}{cccc}1 & 0 & \cdots & 0\end{array}\right] \bar{W}=0} \\
\\
{\left[D_{1}(1,:)\right] \bar{W}=0}\end{array}$ \\
\hline pinned & $\begin{array}{l}{\left[\begin{array}{llll}0 & 0 & \cdots & 1\end{array}\right] \bar{W}=0} \\
{\left[D_{2}(N+1,:)\right] \bar{W}=0}\end{array}$ & $\begin{array}{l}{\left[\begin{array}{cccc}1 & 0 & \cdots & 0\end{array}\right] \bar{W}=0} \\
\\
{\left[D_{2}(1,:)\right] \bar{W}=0}\end{array}$ \\
\hline free & $\begin{array}{c}{\left[D_{2}(N+1,:)\right] \bar{W}=0} \\
S\left(\xi_{N}\right)\left[D_{3}(N+1,:)\right] \bar{W}+S\left(\xi_{N}\right)\left[D_{2}(N+1,:)\right] \bar{W}=0\end{array}$ & $\begin{array}{c}{\left[D_{2}(1,:)\right] \bar{W}=0} \\
S\left(\xi_{0}\right)\left[D_{3}(1,:)\right] \bar{W}+S\left(\xi_{0}\right)\left[D_{2}(1,:)\right] \bar{W}=0\end{array}$ \\
\hline
\end{tabular}

Table 2. Comparison of dimensionless fundamental frequencies of axially FG beams for different values of $\alpha$.

\begin{tabular}{|c|c|c|c|c|c||}
\hline$\alpha$ & source & CF & PP & CP & CC \\
\hline \multirow{4}{*}{-10} & Present solution & 3.5158 & 11.4560 & 16.4022 & 24.0528 \\
\cline { 2 - 6 } & Huang $^{14}$ & 3.5656 & 11.4532 & 16.4775 & 24.0576 \\
\cline { 2 - 6 } & Hein $^{16}$ & NA & 11.4481 & 16.3837 & 24.0269 \\
\cline { 2 - 6 } & Liu $^{23}$ & 3.5340 & 11.4560 & 16.4111 & 24.0660 \\
\hline \multirow{4}{*}{-3} & Present solution $^{16}$ & 3.1409 & 11.2443 & 16.0255 & 23.9433 \\
\cline { 2 - 6 } & Huang $^{14}$ & 3.1421 & 11.2443 & 16.0219 & 23.9456 \\
\cline { 2 - 6 } & Hein $^{16}$ & NA & 11.2422 & 16.0307 & 23.9384 \\
\hline \multirow{4}{*}{3} & Liu $^{23}$ & 3.1410 & 11.2443 & 16.0262 & 23.9446 \\
\cline { 2 - 6 } & Present solution & 2.8545 & 10.3669 & 15.7169 & 24.9364 \\
\cline { 2 - 6 } & Huang $^{14}$ & 2.8544 & 10.3669 & 15.7171 & 24.9375 \\
\cline { 2 - 6 } & Hein $^{16}$ & NA & 10.3670 & 15.7171 & 24.9371 \\
\hline \multirow{3}{*}{ 10 } & Liu $^{23}$ & 2.8545 & 10.3669 & 15.7169 & 24.9366 \\
\cline { 2 - 6 } & Prent solution & 3.0431 & 9.9366 & 15.4957 & 24.8063 \\
\cline { 2 - 6 } & Huang $^{14}$ & 3.0431 & 9.9358 & 15.4956 & 24.7949 \\
\cline { 2 - 6 } & Hein $^{16}$ & NA & 9.9366 & 15.4930 & 24.8080 \\
\hline Liuu $^{23}$ & 3.0431 & 9.9366 & 15.4958 & 24.8068 \\
\hline
\end{tabular}

where $\alpha$ is the material graded index which describes the distribution of material properties. For $\alpha>0$, a larger value $\alpha$ (e.g., 10) represents a more sudden increase in the properties $E(x) / E_{o}$ and $\rho(x) / \rho_{o}$ near the right surface. In contrast, the properties vary abruptly near the left surface for a smaller value of $\alpha$ (e.g., -10 ) as $\alpha<0$. The beam is made of aluminum and zirconia, whose properties vary axially according to the exponential function in Eq. (20). Meanwhile, the beam is aluminum-rich at $x=0$ and zirconia-rich at $x=L$. The dimensionless fundamental natural frequencies $\lambda=\omega L^{2}\left(\rho_{o} A / E_{o} I\right)^{1 / 2}$ of the beams with various values of $\alpha$ and boundary conditions are presented in Table 2 . The present results agree well with those given by Huang and $\mathrm{Li}$, Hein and Feklistova and Liu et al. ${ }^{14,16,23}$ The results reveal that the restraint and material graded index have a significant impact on the frequencies. The fundamental frequency decreases first and increases with the increasing $\alpha$ for the clamped-free $(\mathrm{CF})$ beam, reduces with the increasing $\alpha$ for the pinned-pinned (PP) and clamped-pinned (CP) beam, and varies irregularly with $\alpha$ for the clamped-clamped (CC) beam.

Secondly, a non-uniform axially FG beam is examined whose bending rigidity $S(x)$ and mass per unit length $m(x)$ are represented as: ${ }^{17}$

$$
\begin{aligned}
& S(x)=E(x) I(x)=\left[E_{o}\left(1+\frac{x}{L}\right)\right]\left[\left(1-C_{b} \frac{x}{L}\right)\right. \\
& \left.\left(1-C_{h} \frac{x}{L}\right)^{3} I_{o}\right] ; \\
& m(x)=\rho(x) A(x)=\left[\rho_{o}\left(1+\frac{x}{L}+\left(\frac{x}{L}\right)^{2}\right)\right] \\
& {\left[\left(1-C_{b} \frac{x}{L}\right)\left(1-C_{h} \frac{x}{L}\right) A_{o}\right] .}
\end{aligned}
$$

Here $C_{b}$ and $C_{h}$ denote the width and height taper ratios, respectively, whose values range from 0 to 1 . The non-uniform beam becomes prismatic as $C_{b}=C_{h}=0$, and it tapers to a point at $x=L$ as $C_{b}=C_{h}=1$. The tapered FG beam has the properties of $L=1 \mathrm{~m}, A_{o}=4 \times 10^{-4} \mathrm{~m}^{2}$, $I_{o}=1.33 \times 10^{-8} \mathrm{~m}^{4}, E_{o}=70 \mathrm{Gpa}$ and $\rho=2702 \mathrm{kgm}^{-3}$. Its material properties vary axially according to the polynomial function in Eq. (21). The dimensionless frequency parameter $\lambda=\omega L^{2}\left(\rho_{o} A_{o} / E_{o} I_{o}\right)^{1 / 2}$ is used in the calculations of natural frequencies. Tables $3-5$ present the variations of the dimensionless fundamental frequencies against different taper ratios for axially FG beams with CF, PP and CC boundary conditions, respectively. In comparison with the results obtained by Shahba and Rajasekaran and Liu et al., an excellent agreement is achieved. ${ }^{17,23}$ Depending on the taper ratios and boundary conditions, the increase in height and width taper ratios may decrease or increase the fundamental natural frequencies. For the beams with the same width ratio $C_{b}$, the natural frequencies reduce with the increasing height taper ratio $C_{h}$ except for those of CF beams. The frequencies of CF beams enlarge with the increasing $C_{h}$. For the beams with the same height taper ratio $C_{h}$, the frequencies of $\mathrm{CF}$ beams increase with the increasing width taper ratio $C_{b}$ but those of PP and $\mathrm{CC}$ beams vary differently with $C_{b}$ depending on the value of $C_{h}$. It is important to note that the height taper ratio has a more profound impact on the natural frequencies of all beams than width taper ratio while it shows an opposite trend for CF beams.

From the previous comparison study, it indicates that the proposed method can be applied to evaluate the free vibration frequencies of various axially FG tapered beams with high accuracy. In the next, the free vibration of non-uniform beams with axially graded material properties according to a powerlaw function is studied to show the adaptability of the presented method. Its bending rigidity $S(x)$ and mass per unit length $m(x)$ are given as follows: ${ }^{17,19}$

$$
\begin{aligned}
& S(x)=\left[E_{o}+\left(E_{L}-E_{o}\right)\left(\frac{x}{L}\right)^{p}\right] \\
& {\left[\left(1-C_{b} \frac{x}{L}\right)\left(1-C_{h} \frac{x}{L}\right)^{3} I_{o}\right] ;} \\
& m(x)=\left[\rho_{o}+\left(\rho_{L}-\rho_{o}\right)\left(\frac{x}{L}\right)^{p}\right] \\
& {\left[\left(1-C_{b} \frac{x}{L}\right)\left(1-C_{h} \frac{x}{L}\right) A_{o}\right] .}
\end{aligned}
$$

Here the non-negative exponent $p$ is the volume fraction index. For a larger value of $p$, the properties $E(x) / E_{o}$ and $\rho(x) / \rho_{o}$ change more suddenly near the right surface and the material at the left surface is the dominant constituent. For a smaller value of $p$, the variation of the properties shows an opposite tendency. The recommended value of $p$ ranges from $1 / 3$ to 3 to insure that the FG material has a proper balance between the percentages of the constituents. ${ }^{15}$ The axially FG beam is composed of zirconia and aluminum with ceramic-rich left side and metal-rich right side. The properties of the beam are 
Table 3. Comparison of dimensionless fundamental frequencies of CF axially FG beams with different tapered ratios.

\begin{tabular}{|c|c|c|c|c|c|c|}
\hline$C_{h}$ & source & $C_{b}=0$ & $C_{b}=0.2$ & $C_{b}=0.4$ & $C_{b}=0.6$ & $C_{b}=0.8$ \\
\hline \multirow{3}{*}{0} & Present solution & 2.4256 & 2.6054 & 2.8508 & 3.2137 & 3.8310 \\
\hline & Shahba $^{17}$ & 2.4256 & 2.6054 & 2.8508 & 3.2137 & 3.8310 \\
\hline & $\mathrm{Liu}^{23}$ & 2.4256 & 2.6054 & 2.8508 & 3.2137 & 3.8310 \\
\hline \multirow{3}{*}{0.2} & Present solution & 2.5051 & 2.6863 & 2.9336 & 3.2994 & 3.9219 \\
\hline & Shahba $^{17}$ & 2.5051 & 2.6863 & 2.9336 & 3.2994 & 3.9220 \\
\hline & $\mathrm{Liu}^{23}$ & 2.5051 & 2.6863 & 2.9336 & 3.2993 & 3.9219 \\
\hline \multirow{3}{*}{0.4} & Present solution & 2.6155 & 2.7987 & 3.0486 & 3.4181 & 4.0471 \\
\hline & Shahba $^{17}$ & 2.6155 & 2.7988 & 3.0486 & 3.4181 & 4.0471 \\
\hline & $\mathrm{Liu}^{23}$ & 2.6155 & 2.7987 & 3.0486 & 3.4181 & 4.0471 \\
\hline \multirow{3}{*}{0.6} & Present solution & 2.7835 & 2.9699 & 3.2237 & 3.5985 & 4.2355 \\
\hline & Shahba $^{17}$ & 2.7836 & 2.9699 & 3.2237 & 3.5985 & 4.2355 \\
\hline & $\mathrm{Liu}^{23}$ & 2.7836 & 2.9699 & 3.2237 & 3.5985 & 4.2355 \\
\hline \multirow{3}{*}{0.8} & Present solution & 3.0871 & 3.2794 & 3.5401 & 3.9232 & 4.5695 \\
\hline & Shahba $^{17}$ & 3.0871 & 3.2794 & 3.5401 & 3.9232 & 4.5695 \\
\hline & $\mathrm{Liu}^{23}$ & 3.0871 & 3.2794 & 3.5401 & 3.9232 & 4.5695 \\
\hline
\end{tabular}

Table 4. Comparison of dimensionless fundamental frequencies of PP axially FG beams with different tapered ratios.

\begin{tabular}{|c|c|c|c|c|c|c|}
\hline$C_{h}$ & source & $C_{b}=0$ & $C_{b}=0.2$ & $C_{b}=0.4$ & $C_{b}=0.6$ & $C_{b}=0.8$ \\
\hline \multirow{3}{*}{0} & Present solution & 9.0286 & 9.0599 & 9.0867 & 9.0994 & 9.0685 \\
\hline & Shahba $^{17}$ & 9.0285 & 9.0599 & 9.0867 & 9.0994 & 9.0685 \\
\hline & $\mathrm{Liu}^{23}$ & 9.0286 & 9.0599 & 9.0867 & 9.0994 & 9.0685 \\
\hline \multirow{3}{*}{0.2} & Present solution & 8.1341 & 8.1462 & 8.1498 & 8.1336 & 8.0645 \\
\hline & Shahba $^{17}$ & 8.1341 & 8.1462 & 8.1498 & 8.1336 & 8.0646 \\
\hline & $\mathrm{Liu}^{23}$ & 8.1341 & 8.1462 & 8.1498 & 8.1336 & 8.0646 \\
\hline \multirow{3}{*}{0.4} & Present solution & 7.1530 & 7.1455 & 7.1254 & 7.0794 & 6.9703 \\
\hline & Shahba $^{17}$ & 7.1531 & 7.1455 & 7.1254 & 7.0794 & 6.9703 \\
\hline & $\mathrm{Liu}^{23}$ & 7.1531 & 7.1455 & 7.1254 & 7.0794 & 6.9703 \\
\hline \multirow{3}{*}{0.6} & Present solution & 6.0357 & 6.0082 & 5.9637 & 5.8868 & 5.7351 \\
\hline & Shahba $^{17}$ & 6.0357 & 6.0082 & 5.9637 & 5.8868 & 5.7351 \\
\hline & $\mathrm{Liu}^{23}$ & 6.0357 & 6.0082 & 5.9638 & 5.8868 & 3.7351 \\
\hline \multirow{3}{*}{0.8} & Present solution & 4.6519 & 4.6045 & 4.5353 & 4.4263 & 4.2281 \\
\hline & Shahba $^{17}$ & 4.6520 & 4.6045 & 4.5354 & 4.4263 & 4.2281 \\
\hline & $\mathrm{Liu}^{23}$ & 4.6520 & 4.6046 & 4.5355 & 4.4264 & 4.2284 \\
\hline
\end{tabular}

Table 5. Comparison of dimensionless fundamental frequencies of CC axially FG beams with different tapered ratios.

\begin{tabular}{|c|c|c|c|c|c|c|}
\hline$C_{h}$ & source & $C_{b}=0$ & $C_{b}=0.2$ & $C_{b}=0.4$ & $C_{b}=0.6$ & $C_{b}=0.8$ \\
\hline \multirow{3}{*}{0} & Present solution & 20.4721 & 20.4151 & 20.2883 & 20.0186 & 19.3844 \\
\hline & Shahba ${ }^{17}$ & 20.4721 & 20.4151 & 20.2883 & 20.0186 & 19.3844 \\
\hline & $\mathrm{Liu}^{23}$ & 20.4721 & 20.4152 & 20.2883 & 20.0186 & 19.3845 \\
\hline \multirow{3}{*}{0.2} & Present solution & 18.2170 & 18.1995 & 18.1286 & 17.9436 & 17.4564 \\
\hline & Shahba $^{17}$ & 18.2170 & 18.1995 & 18.1286 & 17.9436 & 17.4564 \\
\hline & $\mathrm{Liu}^{23}$ & 18.2170 & 18.1996 & 18.1286 & 17.9736 & 17.4565 \\
\hline \multirow{3}{*}{0.4} & Present solution & 15.8281 & 15.8497 & 15.8349 & 15.7366 & 15.4021 \\
\hline & Shahba ${ }^{17}$ & 15.8281 & 15.8497 & 15.8349 & 15.7366 & 15.4021 \\
\hline & $\mathrm{Liu}^{23}$ & 15.8282 & 15.8498 & 15.8350 & 15.7367 & 15.4025 \\
\hline \multirow{3}{*}{0.6} & Present solution & 13.2291 & 13.2894 & 13.3316 & 13.3234 & 13.1521 \\
\hline & Shahba $^{17}$ & 13.2291 & 13.2894 & 13.3316 & 13.3234 & 13.1521 \\
\hline & $\mathrm{Liu}^{23}$ & 13.2294 & 13.2896 & 13.3319 & 13.3238 & 13.1529 \\
\hline \multirow{3}{*}{0.8} & Present solution & 10.2217 & 10.3211 & 10.4234 & 10.5143 & 10.5301 \\
\hline & Shahba $^{17}$ & 10.2217 & 10.3211 & 10.4234 & 10.5143 & 10.5301 \\
\hline & $\mathrm{Liu}^{23}$ & 10.2236 & 10.3231 & 10.4257 & 10.5170 & 10.5343 \\
\hline
\end{tabular}

as follows: $E_{o}=200 \mathrm{Gpa}, \rho_{o}=5700 \mathrm{kgm}^{-3}, E_{L}=70 \mathrm{Gpa}$, $\rho_{L}=2702 \mathrm{kgm}^{-3}$, and $r^{2}=I_{o} / A_{o} L^{2}=0.0001 . r$ is the inverse slenderness ratio. The effects of the taper ratios, material graded indices and restraint types on the vibration frequencies of such axially FG beams are demonstrated. The frequency parameter $\lambda=\omega L^{2}\left(\rho_{o} A_{o} / E_{o} I_{o}\right)^{1 / 2}$ is used to evaluate the dimensionless natural frequencies.

Tables 6 and 7 present the effects of various values of $C_{h}$ and $C_{b}$ on the first three dimensionless frequencies for the $\mathrm{CF}$ and PP axially FG beam with $p=2$, respectively. The variations of the first three natural frequencies with respect to the taper ratios $C_{h}$ and $C_{b}$ for the $\mathrm{CP}$ and $\mathrm{CC}$ axially $\mathrm{FG}$ beams are depicted in Figs. 2 and 3 to show the varying trend of the natural frequencies. As seen in Table 6, the first frequency of the $\mathrm{CF}$ beam dramatically increases with the increase of taper ratios $C_{h}$ and $C_{b}$. However, its second and third frequencies reduce with $C_{h}$ but increase with $C_{b}$. It is also noted that the first frequency is affected much more by the increasing width taper ratio $C_{b}$ than the height taper ratio $C_{h}$. In contrast, the height taper ratio has a more profound influence on the higher mode frequencies of the CF beams. As shown in Table 7, the increase in height taper ratio $C_{h}$ has a significant impact on the 


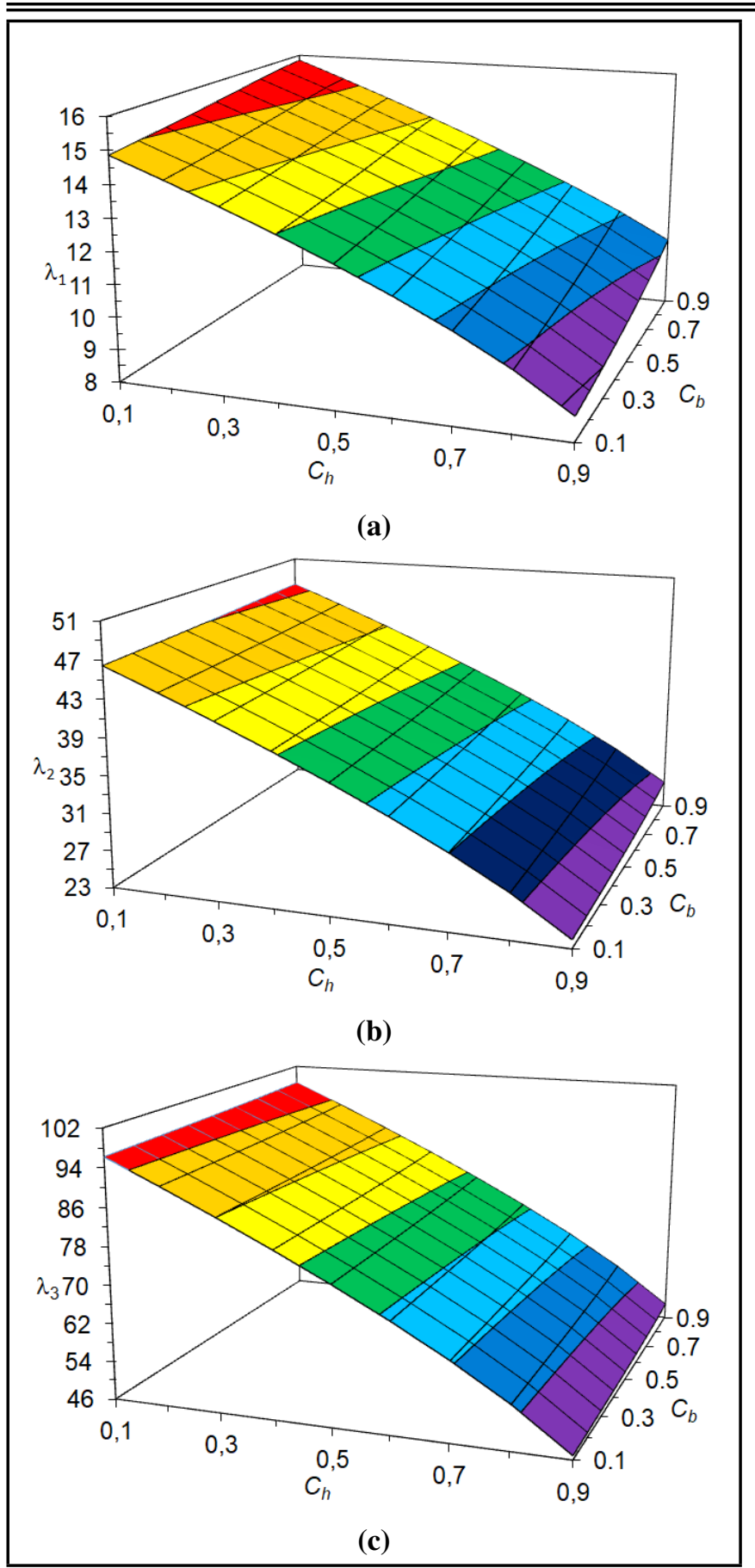

Figure 2. Variation of dimensionless natural frequencies against taper ratios for CP axially FG beams with $p=2$. (a) First mode (b) Second mode (c) Third mode.

reduction of the first three frequencies of the PP beams. The first frequency is most significantly affected by the increasing $C_{h}$, followed by the second and third frequencies. When the width taper ratio $C_{b}$ increases, the first frequency slightly reduces but the second and third frequencies increase. The only exception is the second frequency of the beam with $C_{h}=0.1$, which is decreased as $C_{b}$ is increased from 0.8 to 0.9 . As observed, the height taper ratio will enhance the effect of width taper ratio on the natural frequencies, especially for the first mode. It can be found in Fig. 2 that all three frequencies of $\mathrm{CP}$ beams reduce with the increase in $C_{h}$ and slightly increase with $C_{b}$. Unlike the PP beams, the increasing $C_{h}$ has a more profound effect on the reduction rate of higher mode frequen-

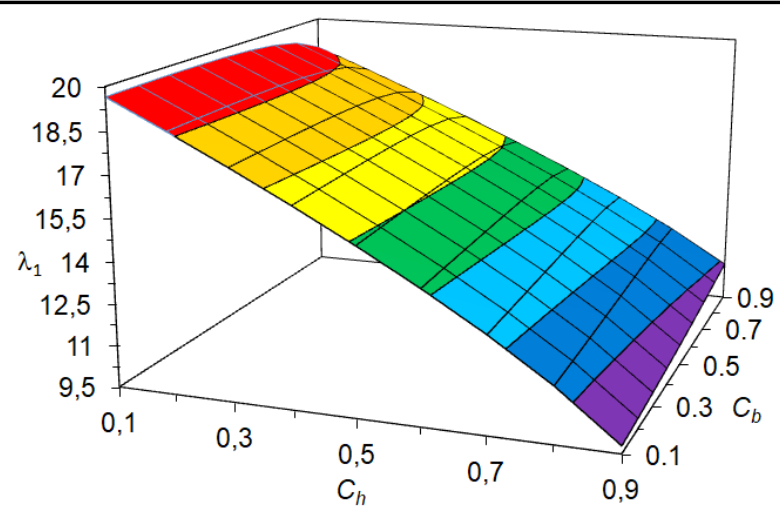

(a)

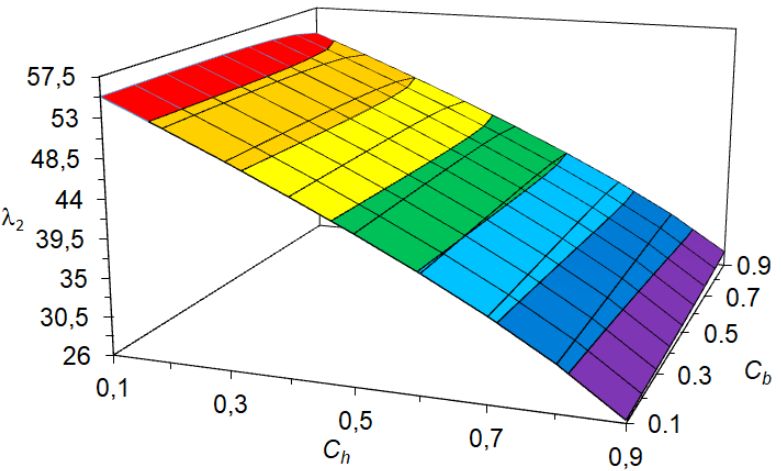

(b)

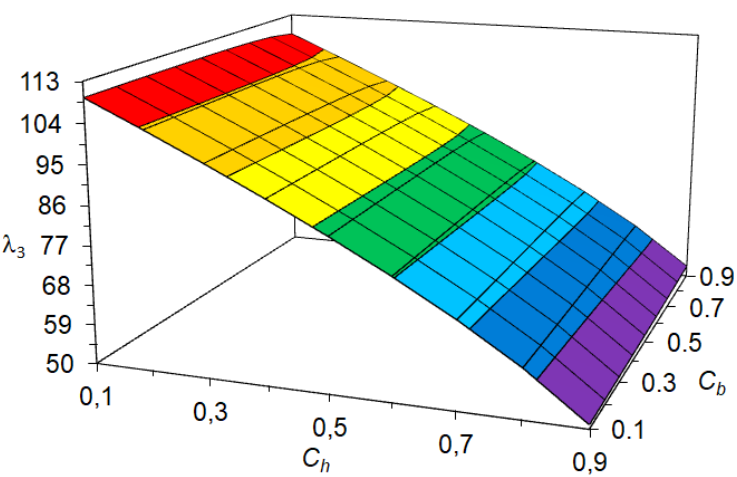

(c)

Figure 3. Variation of dimensionless natural frequencies against taper ratios for CC axially FG beams with $p=2$. (a) First mode (b) Second mode (c) Third mode.

cies. Like the PP and CP beams, Fig. 3 reveals that the first three frequencies of $\mathrm{CC}$ beams decrease as the height taper ratio $C_{h}$ increases. Like the $\mathrm{CP}$ beams, the reduction rate of higher mode frequency with respect to the increasing height taper ratio is higher than that of lower ones. With the increase in $C_{b}$, the first three frequencies of CC beams slightly increase first and then reduce. However, the effect of the increasing $C_{b}$ on the reduction of frequencies is gradually diminished while the $\mathrm{CC}$ beam has a higher height taper ratio $C_{h}$.

To summarize the results reported previously, several conclusions on the effects of taper ratios for axially FG beams with various boundary conditions can be made as follows. In general, the height taper ratio remarkably affects the natural frequencies of all beams than width taper ratio while it shows an opposite tendency for the fundamental frequencies of $\mathrm{CF}$ beams. With the increase in height taper ratio $C_{h}$, the natural frequencies decrease for all beams with the same width ratio 
W.-R. Chen: VIBRATION ANALYSIS OF AXIALLY FUNCTIONALLY GRADED TAPERED EULER-BERNOULLI BEAMS BASED ON CHEBYSHEV...

\begin{tabular}{|c|c|c|c|c|c|c|c|c|c|c|}
\hline$C_{b}$ & mode & $C_{h}=0.1$ & $C_{h}=0.2$ & $C_{h}=0.3$ & $C_{h}=0.4$ & $C_{h}=0.5$ & $C_{h}=0.6$ & $C_{h}=0.7$ & $C_{h}=0.8$ & $C_{h}=0.9$ \\
\hline \multirow{3}{*}{0.1} & $\lambda_{1}$ & 4.4645 & 4.5164 & 4.5773 & 4.6501 & 4.7394 & 4.8524 & 5.0019 & 5.2135 & 5.5488 \\
\hline & $\lambda_{2}$ & 22.6987 & 21.9220 & 21.1204 & 20.2904 & 19.4283 & 18.5303 & 17.5950 & 16.6337 & 15.7326 \\
\hline & $\lambda_{3}$ & 59.3274 & 56.5197 & 53.6280 & 50.6359 & 47.5210 & 44.2511 & 40.7785 & 37.0286 & 32.9061 \\
\hline \multirow{3}{*}{0.2} & $\lambda_{1}$ & 4.6167 & 4.6683 & 4.7288 & 4.8013 & 4.8903 & 5.0029 & 5.1521 & 5.3637 & 5.6998 \\
\hline & $\lambda_{2}$ & 22.9514 & 22.1659 & 21.3552 & 20.5159 & 19.6441 & 18.7360 & 17.7901 & 16.8174 & 15.9038 \\
\hline & $\lambda_{3}$ & 59.5887 & 56.7744 & 53.8757 & 50.8761 & 47.7530 & 44.4740 & 40.9912 & 37.2294 & 33.0918 \\
\hline \multirow{3}{*}{0.3} & $\lambda_{1}$ & 4.7926 & 4.8438 & 4.9040 & 4.9762 & 5.0647 & 5.1769 & 5.3258 & 5.5372 & 5.8737 \\
\hline & $\lambda_{2}$ & 23.2426 & 22.4469 & 21.6258 & 20.7758 & 19.8930 & 18.9735 & 18.0155 & 17.0299 & 16.1022 \\
\hline & $\lambda_{3}$ & 59.8934 & 57.0710 & 54.1636 & 51.1547 & 48.0216 & 44.7317 & 41.2367 & 37.4608 & 33.3060 \\
\hline \multirow{3}{*}{0.4} & $\lambda_{1}$ & 4.9990 & 5.0499 & 5.1098 & 5.1815 & 5.2695 & 5.3813 & 5.5297 & 5.7407 & 6.0772 \\
\hline & $\lambda_{2}$ & 23.5853 & 22.7778 & 21.9446 & 21.0821 & 20.1864 & 19.2535 & 18.2815 & 17.2809 & 16.3372 \\
\hline & $\lambda_{3}$ & 60.2581 & 57.4251 & 54.5067 & 51.4860 & 48.3403 & 45.0369 & 41.5269 & 37.7340 & 33.5586 \\
\hline \multirow{3}{*}{0.5} & $\lambda_{1}$ & 5.2460 & 5.2966 & 5.3560 & 5.4273 & 5.5149 & 5.6261 & 5.7738 & 5.9841 & 6.3200 \\
\hline & $\lambda_{2}$ & 24.0004 & 23.1788 & 22.3311 & 21.4537 & 20.5426 & 19.5937 & 18.6049 & 17.5866 & 16.6240 \\
\hline & $\lambda_{3}$ & 60.7101 & 57.8630 & 54.9298 & 51.8937 & 48.7315 & 45.4105 & 41.8813 & 38.0669 & 33.8664 \\
\hline \multirow{3}{*}{0.6} & $\lambda_{1}$ & 5.5487 & 5.5989 & 5.6580 & 5.7289 & 5.8160 & 5.9266 & 6.0736 & 6.2828 & 6.6168 \\
\hline & $\lambda_{2}$ & 24.5237 & 23.6846 & 22.8189 & 21.9231 & 20.9929 & 20.0242 & 19.0147 & 17.9746 & 16.9890 \\
\hline & $\lambda_{3}$ & 61.2980 & 58.4310 & 55.4772 & 52.4196 & 49.2348 & 45.8896 & 42.3344 & 38.4914 & 34.2583 \\
\hline \multirow{3}{*}{0.7} & $\lambda_{1}$ & 5.9315 & 5.9816 & 6.0405 & 6.1112 & 6.1979 & 6.3079 & 6.4539 & 6.6613 & 6.9914 \\
\hline & $\lambda_{2}$ & 25.2224 & 24.3607 & 23.4718 & 22.5521 & 21.5972 & 20.6028 & 19.5666 & 18.4985 & 17.4837 \\
\hline & $\lambda_{3}$ & 62.1188 & 59.2221 & 56.2375 & 53.1477 & 49.9294 & 46.5488 & 42.9557 & 39.0717 & 34.7933 \\
\hline \multirow{3}{*}{0.8} & $\lambda_{1}$ & 6.4368 & 6.4871 & 6.5463 & 6.6170 & 6.7037 & 6.8133 & 6.9581 & 7.1627 & 7.4852 \\
\hline & $\lambda_{2}$ & 26.2411 & 25.3485 & 24.4279 & 23.4754 & 22.4867 & 21.4573 & 20.3846 & 19.2785 & 18.2240 \\
\hline & $\lambda_{3}$ & 63.4024 & 60.4565 & 57.4211 & 54.2786 & 51.0053 & 47.5669 & 43.9127 & 39.9631 & 35.6144 \\
\hline \multirow{3}{*}{0.9} & $\lambda_{1}$ & 7.1449 & 7.1965 & 7.2569 & 7.3287 & 7.4162 & 7.5259 & 7.6694 & 7.8689 & 8.1755 \\
\hline & $\lambda_{2}$ & 27.9572 & 27.0195 & 26.0526 & 25.0526 & 24.0149 & 22.9350 & 21.8104 & 20.6506 & 19.5378 \\
\hline & $\lambda_{3}$ & 65.8665 & 62.8267 & 59.6945 & 56.4517 & 53.0740 & 49.5264 & 45.7570 & 41.6856 & 37.2102 \\
\hline
\end{tabular}

$C_{b}$ except for the fundamental frequencies of $\mathrm{CF}$ beams. The fundamental frequencies of CF beams show an ascendant tendency with the increasing $C_{h}$. When the width taper ratio $C_{b}$ increases, the first three frequencies increase for $\mathrm{CF}$ and $\mathrm{CP}$ beams with the same height taper ratio $C_{h}$, but those of PP and $\mathrm{CC}$ beams vary differently depending on the value of $C_{h}$. Therefore, it is difficult to predict the natural frequencies for axially FG tapered beams of various boundary conditions with respect to the taper ratios.

Table 8 presents the effects of material gradation on the first three frequencies of axially FG beams with $C_{h}=0.3$ and $C_{b}=0$ under different boundary conditions. As can be seen, the variations of natural frequencies of axially FG tapered beams with respect to $p$ depend on the boundary conditions. With the increasing $p$, the first and second modes of CF beam increase first, and then decrease while the third mode continues to increase. All modes of PP beam enlarge with $p$. For CP and $\mathrm{CC}$ beams, the fundamental frequency increases and decreases alternatively with $p$, while other two modes show an increasing trend. As cited by Shahba et al., it is important to note that the effects of $p$ on the variation of the natural frequencies of tapered axially FG beams are hard to be predicted because both the stiffness and mass of the beam are enhanced with the increase in $p .^{15}$

\section{CONCLUSIONS}

The bending vibration of various axially FG Euler-Bernoulli beams with tapered cross section is studied based on the Chebyshev collocation method. The effects of the material and cross-sectional properties varying along the beam length direction on the vibration behaviors are investigated. Natural frequencies for the uniform axially FG beams with the exponential function gradient and the tapered axially FG beams with polynomial function gradient are evaluated and compared with the published ones to confirm the effectiveness of the present method. The results indicate that the present study can analyse the free vibration of Euler-Bernoulli beams with different axially graded material properties and varying cross-sectional properties under various boundary conditions. Finally, the axially FG tapered beam with power law gradient are examined to demonstrate the adaptability of the present method to different graded material properties. Hence, it is believed that the present method can be extended to study the dynamic problem of elastically supported bi-directional FG beams resting on elastic foundations in the future work.

\section{ACKNOWLEDGEMENT}

This study was funded by the Ministry of Science and Technology of the Republic of China under Grant MOST-1062221-E-034-012.

\section{REFERENCES}

1 REFERENCES Piovan, M. T. and Sampaio, R. A study on the dynamics of rotating beams with functionally graded properties, J. Sound Vib., 327, 134-143, (2009). https://dx.doi.org/10.1016/j.jsv.2009.06.015

2 Simsek, M. Vibration analysis of a functionally graded beam under a moving mass by using different beam theories, Compos. Struct., 92, 904-917, (2010). https://dx.doi.org/10.1016/j.compstruct.2009.09.030

3 Mohanty, S. C., Dash, R. R. and Rout, T. Parametric instability of a functionally graded Timoshenko beam on Winkler's foundation, Nucl. Eng. Des., 241, 2698-2715, (2011). https://dx.doi.org/10.1016/j.nucengdes.2011.05.040 
Table 7. Dimensionless frequencies $\lambda_{i}$ of PP axially FG Z/A beams with different tapered ratios $(p=2)$.

\begin{tabular}{|c|c|c|c|c|c|c|c|c|c|c|}
\hline$C_{b}$ & mode & $C_{h}=0.1$ & $C_{h}=0.2$ & $C_{h}=0.3$ & $C_{h}=0.4$ & $C_{h}=0.5$ & $C_{h}=0.6$ & $C_{h}=0.7$ & $C_{h}=0.8$ & $C_{h}=0.9$ \\
\hline \multirow{3}{*}{0.1} & $\lambda_{1}$ & 9.0338 & 8.4882 & 7.9192 & 7.3217 & 6.6881 & 6.0071 & 5.2595 & 4.4079 & 3.3536 \\
\hline & $\lambda_{2}$ & 36.3792 & 34.4110 & 32.3917 & 30.3101 & 28.1503 & 25.8878 & 23.4819 & 20.8544 & 17.8076 \\
\hline & $\lambda_{3}$ & 81.7246 & 77.2679 & 72.6821 & 67.9385 & 62.9962 & 57.7930 & 52.2253 & 46.0948 & 38.9010 \\
\hline \multirow{3}{*}{0.2} & $\lambda_{1}$ & 9.0096 & 8.4606 & 7.8882 & 7.2875 & 6.6508 & 5.9671 & 5.2171 & 4.3639 & 3.3099 \\
\hline & $\lambda_{2}$ & 36.3993 & 34.4352 & 32.4202 & 30.3430 & 28.1878 & 25.9303 & 23.5300 & 20.9091 & 17.8717 \\
\hline & $\lambda_{3}$ & 81.7592 & 77.3082 & $\begin{array}{l}72.7281 \\
\end{array}$ & 67.9902 & 63.0536 & 57.8562 & 52.2946 & 46.1703 & 38.9835 \\
\hline \multirow{3}{*}{0.3} & $\lambda_{1}$ & 8.9793 & 8.4265 & 7.8506 & 7.2463 & 6.6064 & 5.9196 & 5.1672 & 4.3123 & 3.2586 \\
\hline & $\lambda_{2}$ & 36.4238 & 34.4644 & 32.4543 & 30.3822 & 28.2323 & 25.9806 & 23.5868 & 20.9739 & 17.9481 \\
\hline & $\lambda_{3}$ & 81.8024 & 77.3577 & 72.7839 & 68.0523 & 63.1223 & 57.9315 & 52.3767 & 46.2599 & 39.0816 \\
\hline \multirow{3}{*}{0.4} & $\lambda_{1}$ & 8.9407 & 8.3839 & 7.8041 & 7.1961 & 6.5526 & 5.8626 & 5.1075 & 4.2510 & 3.1977 \\
\hline & $\lambda_{2}$ & 36.4535 & 34.4997 & 32.4952 & 30.4290 & 28.2855 & 26.0407 & 23.6549 & 21.0518 & 18.0408 \\
\hline & $\lambda_{3}$ & 81.8568 & 77.4191 & 72.8525 & 68.1282 & 63.2055 & 58.0224 & 52.4758 & 46.3678 & 39.2002 \\
\hline \multirow{3}{*}{0.5} & $\lambda_{1}$ & 8.8910 & 8.3298 & 7.7457 & 7.1336 & 6.4863 & 5.7928 & 5.0347 & 4.1765 & 3.1240 \\
\hline & $\lambda_{2}$ & 36.4895 & 34.5422 & 32.5444 & 30.4853 & 28.3495 & 26.1132 & 23.7374 & 21.1469 & 18.1551 \\
\hline & $\lambda_{3}$ & 81.9260 & 77.4964 & 72.9379 & 68.2220 & 63.3081 & 58.1341 & 52.5973 & 46.5005 & 39.3467 \\
\hline \multirow{3}{*}{0.6} & $\lambda_{1}$ & 8.8253 & 8.2594 & 7.6706 & 7.0540 & 6.4024 & 5.7051 & 4.9439 & 4.0838 & 3.0323 \\
\hline & $\lambda_{2}$ & 36.5321 & 34.5926 & 32.6031 & 30.5530 & 28.4268 & 26.2015 & 23.8386 & 21.2648 & 18.2992 \\
\hline & $\lambda_{3}$ & 82.0148 & 77.5947 & 73.0460 & 68.3402 & 63.4368 & 58.2742 & 52.7498 & 46.6676 & 39.5327 \\
\hline \multirow{3}{*}{0.7} & $\lambda_{1}$ & 8.7360 & 8.1647 & 7.5708 & 6.9491 & 6.2928 & 5.5912 & 4.8266 & 3.9645 & 2.9141 \\
\hline & $\lambda_{2}$ & 36.5792 & 34.6498 & 32.6711 & 30.6326 & 28.5193 & 26.3087 & 23.9636 & 21.4132 & 18.4855 \\
\hline & $\lambda_{3}$ & 82.1288 & 77.7205 & 73.1841 & 68.4912 & 63.6015 & 58.4538 & 52.9463 & 46.8846 & 39.7778 \\
\hline \multirow{3}{*}{0.8} & $\lambda_{1}$ & 8.6082 & 8.0309 & 7.4311 & 6.8038 & 6.1421 & 5.4356 & 4.6669 & 3.8028 & 2.7527 \\
\hline & $\lambda_{2}$ & 36.6200 & 34.7046 & 32.7408 & 30.7187 & 28.6238 & 26.4346 & 24.1158 & 21.6009 & 18.7330 \\
\hline & $\lambda_{3}$ & 82.2700 & 77.8780 & 73.3586 & 68.6839 & 63.8140 & 58.6883 & 53.2065 & 47.1774 & 40.1165 \\
\hline \multirow{3}{*}{0.9} & $\lambda_{1}$ & 8.4087 & 7.8245 & 7.2176 & 6.5835 & 5.9152 & 5.2025 & 4.4286 & 3.5610 & 2.4988 \\
\hline & $\lambda_{2}$ & 36.6051 & 34.7113 & 32.7710 & 30.7748 & 28.7094 & 26.5550 & 24.2799 & 21.8256 & 19.0716 \\
\hline & $\lambda_{3}$ & 82.4011 & 78.0360 & 73.5454 & 68.9019 & 64.0666 & 58.9805 & 53.5464 & 47.5800 & 40.6019 \\
\hline
\end{tabular}

Table 8. Dimensionless frequencies $\lambda_{i}$ of axially FG Z/A beams with different material graded indices and boundary conditions $\left(C_{h}=0.3, C_{b}=0\right)$.

\begin{tabular}{|c|c|c|c|c|c|c|c|c||}
\hline$B C$ & mode & $p=0.2$ & $p=0.5$ & $p=1$ & $p=2$ & $p=3$ & $p=5$ & $p=10$ \\
\hline \multirow{3}{*}{ CF } & $\lambda_{1}$ & 3.8182 & 4.2269 & 4.4481 & 4.4445 & 4.3455 & 4.1802 & 3.9757 \\
\cline { 2 - 9 } & $\lambda_{2}$ & 18.8631 & 19.8555 & 20.4650 & 20.9133 & 21.1089 & 21.2124 & 21.0340 \\
\cline { 2 - 9 } & $\lambda_{3}$ & 49.1905 & 50.9556 & 52.1801 & 53.4105 & 54.1516 & 54.9396 & 55.3746 \\
\hline \multirow{3}{*}{ PP } & $\lambda_{1}$ & 7.3610 & 7.5499 & 7.7361 & 7.9451 & 8.0613 & 8.1781 & 8.2655 \\
\cline { 2 - 9 } & $\lambda_{2}$ & 29.9177 & 30.8992 & 31.7175 & 32.3676 & 32.6260 & 32.8720 & 33.1385 \\
\cline { 2 - 9 } & $\lambda_{3}$ & 67.2914 & 69.4157 & 71.1670 & 72.6438 & 73.2911 & 73.8883 & 74.4378 \\
\hline \multirow{3}{*}{ CP } & $\lambda_{1}$ & 12.9351 & 13.4722 & 13.6218 & 13.5547 & 13.5187 & 13.5311 & 13.5921 \\
\cline { 2 - 9 } & $\lambda_{2}$ & 39.4242 & 40.6473 & 41.2558 & 41.6533 & 41.8721 & 42.1463 & 42.4794 \\
\cline { 2 - 9 } & $\lambda_{3}$ & 80.8015 & 83.1292 & 84.5982 & 85.8777 & 86.5591 & 87.2573 & 87.9037 \\
\hline \multirow{3}{*}{ CC } & $\lambda_{1}$ & 17.5197 & 17.9293 & 17.8606 & 17.5584 & 17.4236 & 17.3770 & 17.5106 \\
\cline { 2 - 9 } & $\lambda_{2}$ & 47.6082 & 48.7865 & 49.1963 & 49.2651 & 49.2672 & 49.2927 & 49.4399 \\
\cline { 2 - 8 } & $\lambda_{3}$ & 92.7090 & 95.0888 & 96.4150 & 97.3340 & 97.7103 & 97.9841 & 98.1706 \\
\hline \hline
\end{tabular}

4 Thai, H. T. and Vo, T. P. Bending and free vibration of functionally graded beams using various higher-order shear deformation beam theories, Int. J. Mech. Sci., 62, 57-66, (2012). https://dx.doi.org/10.1016/j.ijmecsci.2012.05.014

5 Nguyen, T. K., Vo, T. P. and Thai, H. T. Static and free vibration of axially loaded functionally graded beams based on the first-order shear deformation theory, Compos.: Part B, 55, 147-157, (2013). https://dx.doi.org/10.1016/j.compositesb.2013.06.011

6 Pradhan, K. K. and Chakraverty, S. Effects of different shear deformation theories on free vibration of functionally graded beams, Int. J. Mech. Sci., 82, 149-160, (2014). https://dx.doi.org/10.1016/j.ijmecsci.2014.03.014

$7 \mathrm{Su}, \mathrm{H}$. and Banerjee, J. R. Development of dynamic stiffness method for free vibration of functionally graded Timoshenko beams, Comput. Struct., 147, 107-116, (2015). https://dx.doi.org/10.1016/j.compstruc.2014.10.001

8 Wattanasakulpong, N. and Mao, Q. Dynamic response of
Timoshenko functionally graded beams with classical and non-classical boundary conditions using Chebyshev collocation method, Compos. Struct., 119, 346-354, (2015). https://dx.doi.org/10.1016/j.compstruct.2014.09.004

9 Wattanasakulpong, N. and Chaikittiratana. A. Flexural vibration of imperfect functionally graded beams based on Timoshenko beam theory: Chebyshev collocation method, Meccanica, 50, 1331-1342, (2015). https://dx.doi.org/10.1007/s11012-014-0094-8

10 Wattanasakulpong, N. and Bui, T. Q. Vibration analysis of third-order shear deformable FGM beams with elastic support by Chebyshev collocation method, Int. J. Struct. Stab. Dyna., 18, 1850071, (2018). https://dx.doi.org/10.1142/S0219455418500712

11 Chen, W. R. and Chang, H. Closed-form solutions for free vibration frequencies of functionally graded EulerBernoulli beams, Mech. Compos. Mat., 53, 79-98, (2017). https://dx.doi.org/10.1007/s11029-017-9642-3 
12 Chen, W. R. and Chang, H. Vibration analysis of functionally graded Timoshenko beams, Int. J. Struct. Stab. Dyna., 18, 1850007 (24 pages), (2018). https://dx.doi.org/10.1142/S0219455418500074

13 Aydogdu, M. Semi-inverse method for vibration and buckling of axially functionally graded beams, J. Reinf. Plast. Compos., 27, 683-691, (2008). https://dx.doi.org/10.1177/0731684407081369

14 Huang, Y. and Li, X. F. A new approach for free vibration of axially functionally graded beams with nonuniform cross-section, J. Sound Vib., 329, 2291-2303, (2010). https://dx.doi.org/10.1016/j.jsv.2009.12.029

15 Shahba, A., Attarnejad, R., Marvi, M. T. and Hajilar, S. Free vibration and stability analysis of axially functionally graded tapered Timoshenko beams with classic and non-classical boundary conditions, Compos.: Part B, 42, 801-808, (2011). https://dx.doi.org/10.1016/j.compositesb.2011.01.017

16 Hein, H. and Feklistova, L. Free vibrations of nonuniform and axially functionally graded beams using Haar wavelets, Eng. Struct., 33, 3696-3701, (2011). https://dx.doi.org/10.1016/j.engstruct.2011.08.006

17 Shahba, A. and Rajasekaran, S. Free vibration and stability of tapered Euler-Bernoulli beams made of axially functionally graded materials, Appl. Math. Mod., 36, 3094-3111, (2012). https://dx.doi.org/10.1016/j.apm.2011.09.073

18 Li, X. F., Kang, Y. A. and Wu, J. X. Exact frequency equations of free vibration of exponentially functionally graded beams, Appl. Acoust., 74, 413-420, (2013). https://dx.doi.org/10.1016/j.apacoust.2012.08.003

19 Rajasekaran, S. Differential transformation and differential quadrature methods for centrifugally stiffened axially functionally graded tapered beams, Int. J. Mech. Sci., 74, 15-31, (2013). https://dx.doi.org/10.1016/j.ijmecsci.2013.04.004

20 Huang, Y., Yang, L. E. and Luo, Q. Z. Free vibration of axially functionally graded Timoshenko beams with non-uniform cross-section, Compos.: $\quad$ Part B, 45, 1493-1498, (2013). https://dx.doi.org/10.1016/j.compositesb.2012.09.015

21 Sarkar, K. and Ganguli, R. Closed-form solutions for axially functionally graded Timoshenko beams having uniform cross-section and fixed-fixed boundary condition, Compos.: Part B, 58, 361-370, (2014). https://dx.doi.org/10.1016/j.compositesb.2013.10.077

22 Tang, A. Y., Wu, J. X., Li, X. F. and Lee, K. Y. Exact frequency equations of free vibration of exponentially non-uniform functionally graded Timoshenko beams, Int. J. Mech. Sci., 89, 1-11, (2014). https://dx.doi.org/10.1016/j.ijmecsci.2014.08.017

23 Liu, P., Lin, K., Liu, H. and Qin, R. Free transverse vibration analysis of axially functionally graded tapered Euler-Bernoulli beams through spline finite point method, Shock. Vib., 2016, Article ID 5891030, 23 pages, (2016). https://dx.doi.org/10.1155/2016/5891030
24 Cao, D. and Gao, Y. Free vibration of non-uniform axially functionally graded beams using the asymptotic development method, Appl. Math. Mech., 40, 85-96, (2019). https://dx.doi.org/10.1007/s10483-019-2402-9

25 Celik, I. Approximate computation of eigenvalues with Chebyshev collocation method, Appl. Math. Comput., 168, 125-134, (2005). https://dx.doi.org/10.1016/j.amc.2004.08.024

26 Zhang, X. Mapped barycentric Chebyshev differentiation matrix method for the solution of regular Sturn-Liouville problems, Appl. Math. Comput., 217, 2266-2276, (2010). https://dx.doi.org/10.1016/j.amc.2010.07.027

27 El-Gamel, M. and Sameeh, M. An efficient technique for finding the eigenvalues of fourth-order SturmLiouville problems, Appl. Math., 3, 920-925, (2012). https://dx.doi.org/10.4236/am.2012.38137

28 Saleh Taher, A. H., Malek, A. and Momeni-Masuleh, S. H. Chebyshev differentiation matrices for efficient computation of the eigenvalues of fourth-order Sturm-Liouville problems, Appl. Math. Model., 37, 4634-4642, (2013). https://dx.doi.org/10.1016/j.apm.2012.09.062

29 Hagan, J. and Priede, J. Capacitance matrix technique for avoiding spurious eigenmodes in the solution of hydrodynamic stability problems by Chebyshev collocation method, J. Comput. Phy., 238, 210-216, (2013). https://dx.doi.org/10.1016/j.jcp.2012.12.012

30 Sari, M. S. and Butcher, E. A. Free vibration analysis of non-rotating and rotating Timoshenko beams with damaged boundaries using the Chebyshev collocation method, Int. J. Mech. Sci., 60, 1-11, (2012). https://dx.doi.org/10.1016/j.ijmecsci.2012.03.008

31 Khaneh Masjedi, P. and Maheri, A. Chebyshev collocation method for the free vibration analysis of geometrically exact beams with fully intrinsic formulation, Eur. J. Mech. A-Solid, 66, 329-340, (2017). https://dx.doi.org/10.1016/j.euromechsol.2017.07.014

32 Wattanasakulpong, N. and Mao, Q. Stability and vibration analyses of carbon nanotube-reinforced composite beams with elastic boundary conditions: Chebyshev collocation method, Mech. Adv. Mat. Struct., 24, 260-270, (2017). https://dx.doi.org/10.1080/15376494.2016.1142020

33 Çelik, I. Free vibration of non-uniform Euler-Bernoulli beam under various supporting conditions using Chebyshev wavelet collocation method, Appl. Math. Model., 54, 268280, (2018). https://dx.doi.org/10.1016/j.apm.2017.09.041

34 Rani, R. and Lal, R. Free vibrations of composite sandwich plates by Chebyshev collocation technique, Compos. Part B: Eng., 165, 442-455, (2019). https://dx.doi.org/10.1016/j.compositesb.2019.01.088

35 Trefethen, L. N. Spectral Methods in MATLAB, Software, Environments, and Tools, SIAM, Philadelphia, (2000). 